\title{
Radiation Tolerant Conditioning Electronics for Vacuum Measurements
}

\author{
N. Chatzigeorgiou ${ }^{1}$ \\ CERN \\ Geneva, Switzerland \\ E-mail: nikolaos.chatzigeorgioulcern.ch
}

\section{G. Pigny}

CERN

Geneva, Switzerland

E-mail: gregory.pigny@cern.ch

\section{P. Krakowski}

CERN

Geneva, Switzerland

E-mail: pawel.krakowski@cern.ch

\begin{abstract}
Vacuum in the arcs of the LHC is crucial to minimize beam - gas interactions and to assure thermal insulation of cryostats and helium distribution lines. Several hundred of sensors with their associated conditioning electronics are installed across the arcs for both beam and insulation vacuum measurements. Calculations predict that radiation levels will greatly increase during the HL-LHC era. Therefore, new radiation tolerant conditioning electronics for vacuum measurements are required to withstand such conditions. This paper describes the design of these new electronics, their qualification tests and implementation within the vacuum controls architecture foreseen during the long shutdowns of the LHC.
\end{abstract}

Topical Workshop on Electronics for Particle Physics (TWEPP2018)

17-21 September 2018

Antwerp, Belgium 


\section{Introduction}

In the LHC, about $24 \mathrm{~km}$ of vacuum chambers are located in the Dispersion Suppressors (DS) and arcs areas. Insulation vacuum serves as thermal insulators for cryogenic magnets and helium distribution lines (QRL), with a pressure range from $10^{-6}$ down to $10^{-8}$ mbar by means of turbo-molecular pumping. Beam vacuum minimizes the interaction between the particle beam and any residual air molecules, with a pressure below $10^{-10}$ mbar by means of cryogenic pumping [1]. Three types of pressure sensors with their dedicated conditioning electronics are used to cover the whole pressure range: the membrane, the Pirani and the Penning sensors. Calculations predict that the Total-Ionization-Dose (TID) will reach up to $200 \mathrm{~Gy}$ in the DS and up to $50 \mathrm{~Gy}$ in the arcs by the end of the HL-LHC era [2]. The present system is not designed to withstand such doses. New radiation tolerant electronics has been developed based on Commercial Off-The-Shelf (COTS) components, aiming to withstand 500 Gy with a safety factor of 2.5 for the DS areas.

\section{System Architecture}

\subsection{Present Architecture}

The conditioning electronics of each sensor is deported several meters away from its associated sensor and is located inside a box, on top of the first or second dipole magnet after a quadrupole. This position has been chosen during the LHC buildup to reduce the TID levels on the electronics [3]. Each insulation vacuum sector for cryogenic magnets or QRL has a set of three pressure sensors (membrane, Pirani and Penning). Moreover, the turbo-molecular pumping groups use a set of two pressure sensors (Pirani and Penning). Conditioning electronics transmits analog 0-10V signals to the acquisition system based on Programmable Logic Controller (PLC), located up to $1 \mathrm{~km}$ away, inside a radiation free area. All analog signals are converted to digital pressure reading through 12 bit ADCs. The present position of the conditioning electronics in the tunnel and the absence of modularity make the maintenance difficult. In addition, transmitting 0$10 \mathrm{~V}$ signals over long distances results in increased losses and noise coupling.

\subsection{New Architecture}

The new radiation tolerant electronics will be integrated in a slightly different architecture, mainly to reduce maintenance times, facilitate commissioning campaigns and improve signal integrity. A mini-rack will be installed below the first or second dipole magnet and will house a standard 3U euro-crate. Each sensor will have its own radiation tolerant, modular and hot replaceable conditioning electronic card. The box, which was housing the old electronics, will become a passive patch panel of wires. The connections to the sensors will use the same local cables. The radiation tolerant conditioning electronics will transmit the signals to the PLC in 4$20 \mathrm{~mA}$, avoiding signal losses and reducing noise coupling. Moreover, 4-20mA signal transmissions provide indication in case of broken cables and power cuts. The $3 \mathrm{U}$ euro-crate will be able to house up to nine radiation tolerant conditioning electronic modules, increasing the scalability for potential needs in the future. 


\section{Hardware}

\subsection{Power supply}

An Uninterruptible Power Supply (UPS) will feed the 230VAC to the 3U euro-crate in the tunnel. Two redundant linear AC/DC power supply modules distribute the $\pm 24 \mathrm{VDC} / 2 \mathrm{~A}$ through the backplane of the crate to the conditioning electronic modules. Each conditioning electronic module performs its own regulation from the $\pm 24 \mathrm{VDC}$ bus.

\subsection{Penning Conditioning Electronics}

The Penning sensor relies on the ionization of gas molecules through a cold emission of electrons by using a high electric field between an anode and a cathode. A permanent magnetic field forces spiral trajectories of the electrons in order to increase the probability of ionizing gas molecules [4]. The ionized molecules are collected from the cathode and create an ionization current with a range from $100 \mathrm{uA}$ down to $1 \mathrm{nA}$, which is proportional to pressure (from $10^{-5}$ down to $10^{-9} \mathrm{mbar}$ ). A high voltage of $3 \mathrm{kVDC}$ is generated by a DC/DC converter based on a Royer oscillator, a high frequency voltage set-up transformer and a voltage multiplier bridge. The ionization current from the sensor is converted to voltage by a logarithmic amplifier with a gain of $1 \mathrm{~V} /$ decade. Figure 1 shows the block diagram of the Penning conditioning electronics.

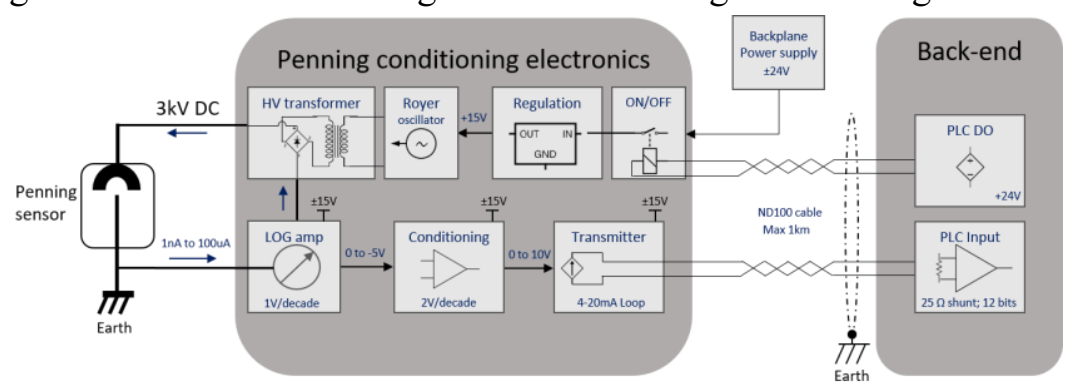

Figure 1- Penning conditioning electronics block diagram

\subsection{Pirani Conditioning Electronics}

Pirani conditioning electronics exploits the pressure dependent thermal conductivity of the gas within a specified pressure range from 1000 mbar down to $10^{-4}$ mbar [4]. A self-powered Wheatstone bridge sources current from $3 \mathrm{~mA}$ to $40 \mathrm{~mA}$ to the tungsten filament of the sensor (which is part of the Wheatstone bridge), while maintaining its temperature to a constant value for the whole pressure range. The output voltage of the bridge is a non-linear function of the pressure. Conditioning stages are used for calibration at low and atmospheric pressures, linearization and scaling. Figure 2 shows the block diagram of the Pirani conditioning electronics.

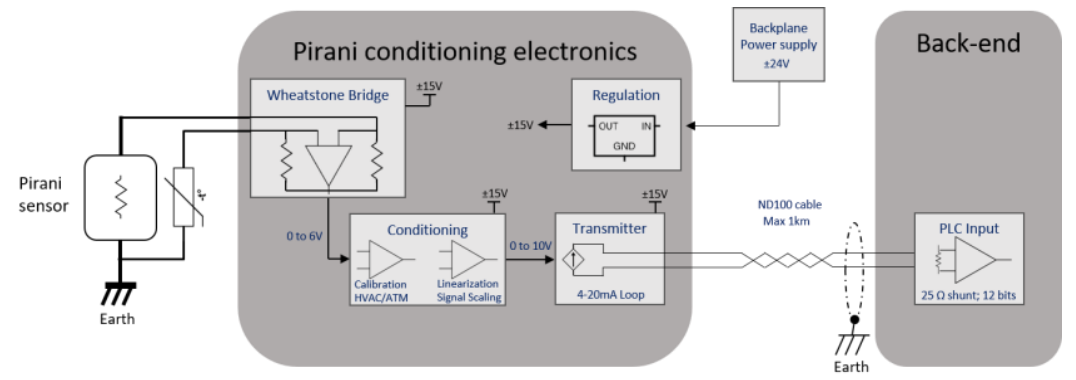

Figure 2- Pirani conditioning electornics block diagram 


\subsection{Membrane Conditioning Electronics}

The piezo-resistive sensor is composed by a thin silicon crystal layer used as a membrane, whose deformation induces a piezo-resistive effect on the four resistances of a Wheatstone bridge [4]. An integrated amplifier circuitry is connected to the bridge, providing a $0-10 \mathrm{~V}$ output signal corresponding to a pressure range from $1 \mathrm{mbar}$ up to $1600 \mathrm{mbar}$. The sensor requires a regulated supply of $\pm 13.5 \mathrm{~V}$. Figure 3 shows the block diagram of the membrane conditioning electronics.

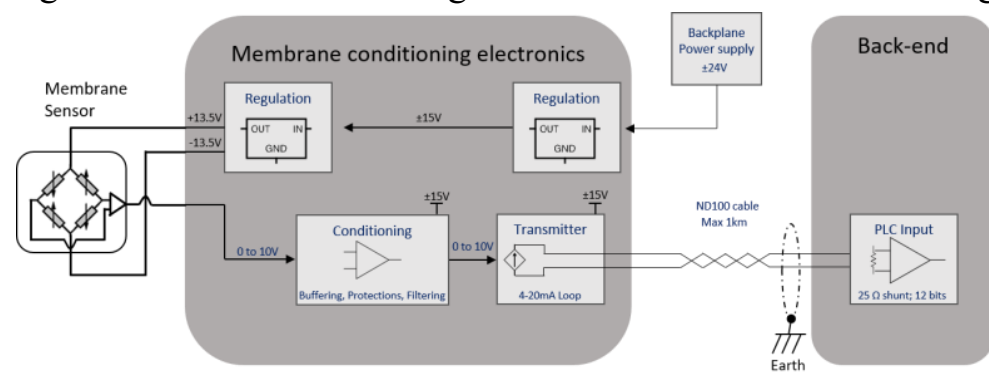

Figure 3 - Membrane conditioning electronics block diagram

\section{Testing and Qualifications}

\subsection{COTS Components Radiation Screening}

The conditioning electronics and their tolerance to radiation are based on COTS components. After the conceptual design, 18 components fulfilling the design requirements have been selected for component level radiation tests. Those tests have been carried out in the PaulScherrer-Institute (PSI) in Villigen, Switzerland. Components have been exposed to a $200 \mathrm{MeV}$ proton beam with a dose rate of $350 \mathrm{~Gy} / \mathrm{h}$ and High Energy Hadron (HEH) fluence of $10^{12} \mathrm{~cm}^{-2}$. All the components accumulated up to $500 \mathrm{~Gy}$ and depending on the component type, power cycles were performed every $50 \mathrm{~Gy}$ or $250 \mathrm{~Gy}$ in order to study any potential latch-up. 3 noncritical components out of 18 were rejected; they were potential candidates of qualified components.

\subsection{System-Level Characterization in non-radiative environment}

Specific test benches were used for each conditioner to characterize their response to simulated pressure, by using Source Meter Units (SMU) at room temperature. Long-term stability tests have been performed over several days. Then, the conditioners were characterized with a real pressure measurement at room temperature, connected to their respective sensors mounted on a vacuum chamber, in which the pressure could range accordingly. The resulting pressure reading from the conditioning electronics was compared to a reference pressure sensor, which was mounted on the same vacuum chamber. In addition, the conditioners have been tested in a climatic chamber. The temperature inside the climatic chamber swept from 5 to $70{ }^{\circ} \mathrm{C}$ with $5{ }^{\circ} \mathrm{C}$ steps.

\subsection{System-Level Characterization in radiative environment}

The system level qualification under ionizing radiations has been performed at the CERN High energy Accelerator Mixed field facility (CHARM). The conditioning electronics were irradiated with secondary particles of wide energy spectra, up to $24 \mathrm{GeV}$ and fluence of $2.10^{12}$ $\mathrm{cm}^{-2}$. Four campaigns took place, one per conditioner. Each of the campaigns lasted for 2 weeks 
with an approximate dose rate of $2 \mathrm{~Gy} / \mathrm{h}$. All the electronics successfully accumulated $500 \mathrm{~Gy}$ with measurement errors within tolerable margins. The $3 \mathrm{kVDC}$ high voltage of the Penning electronics deviated by $2 \%$, assuring a proper discharge current in the sensor, while the pressure readout error was kept below $6 \%$. The Pirani conditioning electronics introduced a $2.5 \%$ pressure readout error while they could properly bias the filament of the sensor. The regulated $\pm 13.5 \mathrm{~V}$ supplies for the membrane sensor deviated by $0.1 \%$ and the pressure readout error was kept below $3 \%$. The $\pm 24 \mathrm{~V}$ from the power supply card deviated by $2 \%$, assuring enough supply voltage to the conditioning electronics to perform their own regulations.

\subsection{Control System}

A dedicated control system based on PLC and Supervisory Control And Data Acquisition (SCADA) using WinCC OA has been developed for this purpose. The hardware part is divided in four different euro-crates. The first crate houses the PLC CPU, assures the communications with the SCADA, controls up to three SMUs and provides 16 digital outputs. A second crate is dedicated to acquire up to 24 temperature measurements through PT100 sensors with 12 bits resolution. The third crate is responsible for analog signal measurements and can sample up to 56 voltage and 8 current channels every second with 16 bits resolution. The last crate is used as voltage source unit and can source up to 8 analogue channels with 12 bits resolution. The user can read all the analog values through a drop-down list, access to the digital outputs and can remotely control the conditioning electronics. For each analog value, the user can set alarms, which can trigger notification emails. Moreover, the user has visual information concerning critical parts of the control system such as the status of the hardware, the archive manager and the peripherals. Lastly, an export panel allows the user to define dates, times and the desired channels to export.

\section{Conclusion}

The new architecture of the vacuum measurement electronics will greatly decrease maintenance and commissioning times, while it will enhance signal integrity and provide smooth integration within the vacuum controls system. The characterization of the conditioning electronics meets the required specifications under the different test environments. Regarding the tolerance to radiation, the new conditioning electronics can withstand doses up to $500 \mathrm{~Gy}$ with satisfying performance degradation. They will be replacing the present electronics in the DS areas during the second long-shutdown (LS2) and in the arcs during the third long-shutdown (LS3).

\section{References}

[1] P. Gomes et al., "The Control System of CERN Accelerators Vacuum”, ICALEPCS11, Grenoble, 2011.

[2] R. Garcia Alia et al., "LHC and HL-LHC: Present and Future Radiation Environment in the HighLuminosity Collision Points and RHA Implications”, IEEE Transactions on Nuclear Science, 2017.

[3] P. Krakowski et al., "R2E of critical Vacuum Controls in the LHC", RADECS16, Bremen, 2016.

[4] G. Pigny et al., "Measurements, Alarms and Interlocks in the Vacuum Control System of the LHC", ICALEPCS15, Melbourne, 2015. 